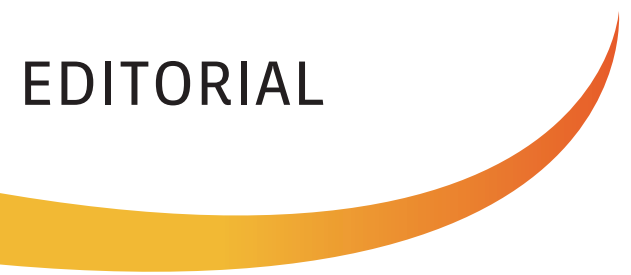

Referência

\title{
Health literacy: A nursing perspective
}

Literacia em saúde: A perspetiva da enfermagem

Graeme D. Smith ${ }^{1}$

iD https://orcid.org/0000-0003-2974-3919

${ }^{1}$ Caritas Institute of Higher Education, Hong Kong
Corresponding author

Graeme D. Smith

E-mail: smithgd0901@gmail.com
Nursing plays a key role in maintaining and protecting global health. With a workforce of over 28 million, nursing represents by far the largest group of health care workers (World Health Organization [WHO], 2020). The value of nursing has become inherently clear during the COVID-19 pandemic, as nurses worldwide have made a strong contribution as health care providers, educators, researchers, and patient advocates (Smith et al., 2020). At a time of pandemic, nurses have been required to provide accurate health-related information to patients in an understandable way. Despite increasing awareness of the importance of providing health-related information across the entire health care continuum, it is perhaps surprising to discover that limited attention has been given to the concept of health literacy in the nursing literature. In recent years, health literacy has evolved from its original focus on elementary literacy skills, and it is now widely recognized to include the ability to critically analyze and use health-related information, providing the capacity to engage well with healthcare systems. From a health provider's perspective, health literacy can be defined as "linked to literacy and entails people's knowledge, motivation and competencies to access, understand, appraise, and apply health information to make judgments and take decisions in everyday life concerning healthcare, disease prevention and health promotion to maintain or improve quality of life during the life course" (Sørensen et al., 2012, p. 3). As such, it relates to the ability of someone to access, understand, appraise, and then apply health-related information (Sørensen et al., 2015). When dealing with health literacy issues, it is also important for nurses to understand that nobody is ever fully health literate. Everyone requires some help to navigate complex health-related situations, as evidenced by the outbreak of the COVID-19 pandemic (Jackson et al., 2020). Therefore, adequate health literacy does not only apply to the ability to read and comprehend health-related information. Ultimately it relates to people's ability to take more responsibility for their health. In this editorial, by examining the current evidence, I aim to highlight the importance of health literacy in nursing, making specific reference to the pandemic, stressing the importance of health literacy in nurse education, underlining a potential link that may exist between health literacy, resilience, and empowerment.

Limited health literacy may be a greatly underestimated predicament in the general population worldwide. The WHO has indicated that health literacy is generally low in developed and developing countries (WHO, 2016). Sørensen et al. (2015) reported that up to half of all European adults had some form of health literacy limitation. Despite this, nurses are commonly known to overestimate patients' level of health literacy. Therefore, regardless of geographical location or culture, it can be assumed that people may have issues understanding health-related information given to them by nurses. When any form of health-related information is created that is difficult to understand, potential health literacy problems will arise.

The nursing profession is ideally positioned to help and support those with limited health literacy, including older adults, and the potential benefits of enhanced health 
literacy in people are enormous (Smith et al., 2021a). Importantly, nurses' delivery of shame-free health literacy support has been associated with greater levels of empowerment, engagement, activation, and optimal health-related outcomes (Loan et al., 2018). Regardless of the nursing specialty, health literacy is an essential skill to enhance effective communication and successfully facilitate person-centered care.

Health literacy is an extremely complex concept. Patients with lower levels of health literacy have been associated with sub-optimal health and health outcomes, such as compliance with treatment regimes, medication adherence, and the ability for self-management (Smith et al., 2021b). Low health literacy may be linked to poorer health status and impairment to quality of life. The WHO believed health literacy to be a better predictor of health than many other social determinants of health including, ethnicity, education, and employment status (Kickbusch et al., 2013). Indeed, if not one of the most persuasive forecasters of health, health literacy has become the strongest social determinant of health (Ho \& Smith 2020).

The global importance of health literacy has been exemplified by the COVID-19 global pandemic (Paakkari \& Okan, 2020). The rapid arrival of COVID-19 required people to adapt very quickly to acquire and apply health-related information, often with drastic modifications to lifestyle and health-related behavior. Notwithstanding the enormous humanitarian and economic consequences of COVID, there have been ongoing challenges with information accuracy and overload. The COVID-19 outbreak demonstrates the care that needs to be taken with the reliability and dependability of health-related information. Sentell et al. (2020) believed that the global pandemic provided a crucial test for health literacy, highlighting its importance in population health and well-being. Governments and health-providing agencies worldwide appear to be increasingly aware that population-based enhancement of health literacy may be equated with decreased health inequalities and improved health outcomes.

Regardless of the caring environment, the nursing staff is an important provider of patient education and health-related information. Therefore, it is somewhat surprising that although the nursing profession shoulders most of the responsibility for delivering health-related patient education, limited global attention has been given to nursing education of health literacy.

Over a decade ago, Scheckel et al. (2010) highlighted the importance of health literacy education, specifically advocating more innovative approaches to teaching health literacy in nursing. Enhanced education in the practice of health literacy may expand nursing students' existing skills and knowledge. However, few educational institutions subscribe to offering health literacy nurse education. Within many nursing curricula, the preparation of nursing students to be equipped with an awareness of health literacy appears to have been neglected. A good starting point and potential solution could be the inclusion of health literacy-related questions in all formats of nursing assessment. Nursing students should also be encouraged to ask open-ended questions and use 'teach-back' methods when providing health-related information to people. Using the 'teach-back' approach helps confirm that the nurse has provided information in an understandable format because patients' understanding can be verified when they repeat the information in their own words. The value of the 'teach-back' approach is that it can empower nurses to confirm patient understanding and correct any erroneous information.

Despite the development of several promising nursing health literacy initiatives in recent years, regrettably, these appear to have been grossly under-utilized by the profession (Loan et al., 2018). Universal health literacy education for all undergraduate nursing students, based upon sound evidence, can be the best way forward to improve the situation, easing widespread literacy problems. From a post-registration nursing perspective, Parnell and Agris (2020) have argued that the development of strategic competency-based health literacy education may enhance the health literacy professionalism of nurses. Due to the fast-changing and dynamic nature of health literacy, these educational initiatives are required as a matter of urgency.

Around the world, nurses should not only be aware of the potential problems associated with health illiteracy, but they should also be able to actively try to remedy the situation. Nurses advocate a universal precautions approach to health literacy, which assumes that all patients may have difficulty understanding health-related information. Unlike other socio-demographic characteristics that people may have, like age and ethnicity, which are fixed, reasonable evidence suggests that health literacy levels can be modified and enhanced (Nutbeam, 2000). Berkman (2011) demonstrated that health literacy interventions can improve patients' comprehension of health-related information. To date, health literacy interventions have mainly focused on improving health literacy skills via improved patient-healthcare professional communication, simplifying health-related educational materials, and eHealth interventions. To enhance the effectiveness of health literacy nursing interventions, they must undergo appropriate testing, have a strong basis in theory, and place a strong emphasis on skill-building.

With health literacy interventions comes the need for evaluation, which requires health literacy measurement tools. Traditionally the measurement of health literacy has been known to be tricky. In their assessment of health literacy in older adults, Eronen et al. (2019) highlighted the many challenges that nurse researchers can face when measuring this critical determinant of health. Historically, researchers have applied objective measures, like the REALM, when researching older adults (Kobayashi et al., 2016). Subsequently, comprehensive subjective measurement tools have begun to emerge. One of these, the HLS-EU, originally developed and validated in Europe, is now used worldwide. The HLS-EU provides a scale focused on task-specific competencies, such as illness prevention and health promotion. The WHO has strongly advocated the important role of health literacy in developing empowerment and resilience 
(Kickbusch et al., 2013).

In this sense, empowerment can be seen as a process through which people gain more control over their lives, health, and determinants. Through empowerment, health literacy programs contribute to democratizing the health care system and achieving a stronger commitment to health and well-being in communities and society. Resilience can be seen as a form of adaptation, recovery, and bouncing back in the face of adversity. Health literacy may be viewed as an asset for individuals and communities within both perspectives. As such, any investment made to strengthen health literacy may yield a substantial return in enhanced health and well-being (Abel \& Frohlich, 2021). Combined with appropriate social resources, health literacy can become a valuable asset to help people become more empowered and resilient.

Health literacy is a vital but often overlooked concept that can enhance the patient experience. This editorial aimed to capture some topical aspects of health literacy in relation to nursing. Globally, health literacy has become one of the most important determinants of health. It can improve health outcomes and prove crucial in developing resilience and empowerment in the general population. COVID-19 has highlighted the importance of health literacy worldwide. More consideration of the management, research and education of this important social determinant of health in the nursing profession is deserved and urgently required. To ensure the effective delivery of evidence-based health literacy practice, high-quality research is essential, and nurses are in an optimal position to conduct this research.

\section{References}

Abel, T., \& Frohlich, K. L. (2012). Capitals and capabilities: Linking structure and agency to reduce health inequalities. Social Science and Medicine, 74(2), 236-244. https://doi.org/10.1016/j.socscimed.2011.10.028

Berkman, N. D., Sheridan, S. L., Donahue, K. E., Halpern, D. J., \& Crotty, K. (2011). Low health literacy and health outcomes: An updated systematic review. Annals of Internal Medicine, 155(2), 97-107. https://doi.org/10.7326/0003-4819-155-2-201107190-00005

Eronen, J., Paakkari, L., Portegijs, E., Saajanaho, M., \& Rantanen, T. (2019). Assessment of health literacy among older Finns. Aging Clinical and Experimental Research, 31(4), 549-556. https://doi.org/10.1007/s40520-018-1104-9

Ho, K. H., \& Smith, G. D. (2020). A discursive paper on the importance of health literacy among foreign domestic workers during outbreaks of communicable diseases. Journal of Clinical Nursing, 29(23-24), 4827-4833. https://doi.org/10.1111/jocn.15495

Jackson, D., Bradbury-Jones, C., Baptiste, D., Gelling, L., Morin, K., Neville, S., \& Smith, G. D. (2020). Life in the pandemic: Some reflections on nursing in the context of COVID-19. Journal of Clinical Nursing, 29(13-14), 2041-2043. https://doi.org/10.1111/jocn.15257

Kickbusch, I., Pelikan, J. M., Apfel, F., \& Tsouros, A. (Eds.). (2013). Health literacy: The solid facts. World Health Organization. https://apps. who.int/iris/bitstream/handle/10665/128703/e96854.pdf

Kobayashi, L., Wardle, J., Wolf, M., \& von Wagner, C. (2016). Aging and functional health literacy: A systematic review and meta-analysis. The Journals of Gerontology: Series B, Psychological Sciences and Social Sciences, 71(3), 445-457. https://doi.org/10.1093/geronb/gbu161

Loan, L. A., Parnell, T. A., Stichler, J. F., Boyle, D. K., Allen, P., VanFosson, C. A., \& Barton, A. J. (2018). Call for action: Nurses must play a critical role to enhance health literacy. Nursing Outlook, 66(1), 97-100. https://doi.org/10.1016/j.outlook.2017.11.003

Nutbeam, D. (2000). Health literacy as a public health goal: A challenge for contemporary health education and communication strategies into the 21st century. Health Promotion International, 15(3), 259-267. https://doi.org/10.1093/heapro/15.3.259

Paakkari, L., \& Okan, O. (2020). COVID-19: Health literacy is an underestimated problem. Lancet Public Health, 5(5), e249-e250. https:// doi.org/10.1016/s2468-2667(20)30086-4

Parnell, T. A., \& Agris, J. (2020). Health literacy: An essential element of health care professionalism and resilience. Studies in Health Technology and Informatics, 269, 65-71. https://doi.org/10.3233/shti200023

Scheckel, M., Emery, N., \& Nosek, C. (2010). Addressing health literacy: The experiences of undergraduate nursing students. Journal of Clinical Nursing, 19(5-6), 794-802. https://doi.org/10.1111/j.1365-2702.2009.02991.x

Sentell, T., Vamos, S., \& Okan, O. (2020). Interdisciplinary perspectives on health literacy research around the world: More important than ever in a time of COVID-19. International Journal of Environmental Research and Public Health, 17(9), 3010. https://doi.org/10.3390/ ijerph17093010

Smith, G. D., Ng, F., \& Li, W. H. (2020). COVID-19: Emerging compassion, courage and resilience in the face of misinformation and adversity. Journal of Clinical Nursing, 29(9-10), 1425-1428. https://doi.org/10.1111/jocn.15231

Smith, G. D., Ho, K. H., Poon, S., \& Wai-Chin, S. (2021a). Beyond the tip of the iceberg: Health literacy in older people [Editorial]. Journal of Clinical Nursing. Advance Online Publication. https://doi.org/10.1111/jocn.16109

Smith, G. D., Lai, V., \& Poon, S. (2021b). Building the case for health literacy in gastroenterology. Gastrointestinal Nursing, 19(7), 26-30. https://doi.org/10.12968/gasn.2021.19.7.26

Sørensen, K., Van den Broucke, S., Fullam, J., Doyle, G., Pelikan, J., Slonska, Z., Brand, H., \& (HLS-EU) Consortium Health Literacy Project European. (2012). Health literacy and public health: A systematic review and integration of definitions and models. BMC Public Health, 12(80). https://doi.org/10.1186/1471-2458-12-80

Sørensen, K., Pelikan, J. M., Röthlin, F., Ganahl, K., Slonska, Z., Doyle, G., Fullam, J., Kondilis, B., Agrafiotis, D., Uiters, E., Falcon, M., Mensing, M., Tchamov, K., van den Broucke, S., \& Brand, H., \& HLS-EU Consortium. (2015). Health literacy in Europe: Comparative results of the European health literacy survey (HLS-EU). European Journal of Public Health, 25(6), 1053-1058. https://doi.org/10.1093/ eurpub/ckv043 
World Health Organization. (2016, November 21-24). Policy brief 4: Health literacy [Policy brief]. Promoting health in the Sustainable Development Goals: Health for all and all for health. The $9^{\text {th }}$ Global Conference on Health Promotion, Shanghai, China. https://www.who.int/ healthpromotion/conferences/9gchp/policy-brief4-health-literacy.pdf?ua=1

World Health Organization. (2020). State of the World's Nursing 2020: Investing in education, jobs and leadership. https://www.who.int/publications/i/item/9789240003279 